\title{
Scaling Relations for Dislocation Structure and Response
}

\author{
R. LeSar ${ }^{* 1}{ }^{1}$ M. Koslowski, ${ }^{2}$ Robb Thomson, ${ }^{3}$ and J. M. Rickman ${ }^{4 \dagger}$ \\ ${ }^{1}$ Theoretical Division, Los Alamos National Laboratory, Los Alamos, NM 87545 USA \\ ${ }^{2}$ Department of Mechanical Engineering, Purdue University, West Lafayette, IN 47907 USA \\ ${ }^{3}$ (Retired) 250 E. Alameda, Apt 515, Santa Fe, NM 87501 USA \\ ${ }^{4}$ Department of Materials Science and Engineering and Department of Physics, Lehigh \\ University, Bethlehem, PA 18015 USA \\ E-mail:lesarelanl.gov
}

\begin{abstract}
A major goal in dislocation theory is the development of a coarse-grained method that would enable predictions of dislocation response without resolving the degrees of freedom of all the dislocations. While a number of possible coarse-grained theories have been proposed, all of these need information about structures at scales smaller than the coarse-graining volume. Here we present results from dislocation simulations that yield scaling relations with the hope that they can provide a framework for modeling the sub-scale dislocation structures and dynamics. For example, we show that dislocations have a self-similar (fractal) structure over wide range of stress/strain; dislocations move by means of avalanches that have power-law (self-organized critical) behavior; and the energy, order and noise all scale as powers of the density, with a welldefined balance between noise and order.
\end{abstract}

International Conference on Statistical Mechanics of Plasticity and Related Instabilities

31 August - 2 September 2005

Bangalore, India

\footnotetext{
* Speaker.

${ }^{\dagger}$ The work of R. LeSar and M. Koslowski was performed under the auspices of the United States Department of Energy (US DOE under contract W-7405-ENG-36) and was supported by the Division of Materials Science of the Office of Basic Energy Sciences of the Office of Science of the US DOE. MK thanks the Computational Materials Science Network of the US DOE/OS/OBES/DMS for their support. JMR thanks the Air Force Office of Scientific Research for their support under grant FA9550-05-1-0082.
} 


\section{Introduction}

Plastic deformation in crystalline metals is a consequence of the motion of large numbers of dislocations. In a deformable material, such as a metal, typical dislocation densities are around $10^{13}$ to $10^{15} / \mathrm{m}^{2}$, i.e., $10^{13}$ to $10^{15}$ meters of dislocations in a cubic meter of material. The mobility of dislocations gives rise to plastic flow at relatively low stress levels compared to the theoretical strength. Dislocations also form organized structures such as walls, cells and pile-ups. Differences owing to the topological constraints placed by crystallography greatly add to the complexity of describing dislocation microstructural evolution and dynamics.

We know from experiments that despite the complexity

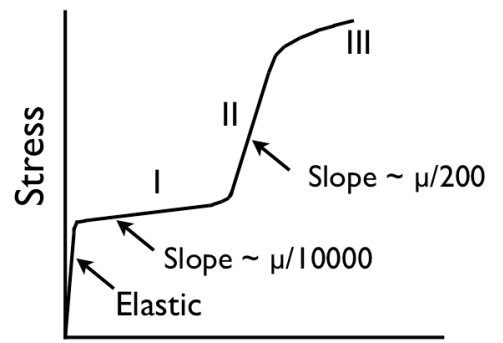

Strain

Figure 1: Schematic stress-strain curve for an $f c c$ single crystal under single-slip conditions. of dislocation structure and dynamics, it is possible to describe the overall deformation behavior of a material with relatively few variables. For example, in Figure 1 we show a typical stress-strain curve for fcc single crystals such as copper, aluminum, etc. under single slip loading conditions.(1) The response can be described in terms of distinct regions (Stages), each having a corresponding unique behavior. Indeed, the slopes of the stress-strain curves (the hardening rate) in each linear region (Stage I and II) are "universal" factors of the shear modulus $\mu$. The general features of the dislocation microstructures in each stage have also been characterized.(2-4) The dislocation patterns are not ordered, but show evidence of both randomness and heterogeneity.

The development of scaling relations provides a useful approach to describing systems with multiscale properties. As an example, consider the deformation of $f c c$ materials. At high enough strain, a cellular structure develops, in which regions with low dislocation density are surrounded by relatively thin, high-density, walls. A standard approach for characterizing such structures is to analyze their fractal characteristics. Fractal systems are self-similar; structures at one scale appear the same (at least in a statistical sense) as structures at other scales. Thus, they can be characterized by correlation functions in the form of power laws.

A number of studies have been undertaken to determine whether dislocation patterns are, indeed, fractal, starting with detailed computer simulations that yielded fractal dislocation patterns.(5) Hahner and coworkers (6) later characterized experimental dislocation structures under large deformation and found similar fractal behavior. Szekeley et al.(4) found a strong correlation between the fractal dimension and the relative dislocation density variance described above. Zaiser et al. recently analyzed the surface morphology of metals after plastic deformation over a range of scales and found that the results could be understood in terms of a fractal distribution of plastic strain within the deformed samples.(7)

To this point, we have discussed the static properties of dislocations in strain hardening. Another important characterization of the dislocation response measures the inherently stochastic nature of the dislocation motion. Crystalline materials subjected to an external stress display bursts of activity owing to nucleation and motion of dislocations.(8-11) Sudden local changes during plastic deformation generate acoustic emission waves that reveal the intermittent and jerky character 
of dislocation motion. Acoustic emission experiments on single crystals of ice under viscoplastic deformation (creep) show that the probability density function of the acoustic emission intensity, $P(A)$, follows a power law distribution $P(A) \sim A^{-\sigma}$ with an exponent $\sigma \sim 1.6-2.0$ (12). Selfsimilarity in dislocation avalanches was also observed spatially, that is, the probability of two avalanches being separated by a distance less than $r$ is given by $C(r) \sim r^{D}$ with $D=2.5 \pm 0.1$.(13) Although many acoustic emission measurements have been reported, only qualitative descriptions of the relation with plastic deformation have be made. Recently, a direct measurement of intermittent behavior in dislocation flow has been measured in single crystals of $\mathrm{Ni}$, yielding power-law scaling with an exponent $\sigma=1.60 \pm 0.02$.(14)

One question that arises from the avalanche behavior is whether dislocation response exhibits self-organized criticality. $(10 ; 12 ; 15)$ The notion of self-organized criticality (SOC) was introduced by Bak et al.(16) to explain systems that organize themselves into a (stationary) critical state in which a minor event can start a chain reaction that can have an effect over a large scale. The critical state is characterized by correlation functions that follow power laws.(17) The importance of SOC theory arises from its ability to explain a wide set of diverse, and seemingly unrelated phenomena, such as earthquakes, fracture, dynamics of magnetic domains, etc.(18) There is no rigorous definition or mathematical formalism of self-organized critical behavior; SOC is a phenomenological definition. It can be characterized by (i) power law distributions without any apparent tuning; (ii) the process connected with the external driving of the system needs to be much slower than the internal relaxation processes; (iii) the presence of marginally stable states (Bak et al (16) view marginally stable states as those characterized by the lack of any typical length or time scale), related to systems at a critical point); and (iv) a very large number of interacting entities.(19) If dislocations are self-organized critical systems, then we can bring to bear on deformation another important type of scaling and analysis. A brief description of SOC in deformation has recently been published.(20)

Over the past fifty years, numerous models have been introduced to try to describe the complex patterning and response in systems of dislocations, including a number of stochastic theories that attempt to interpret the process of pattern formation during the Stage II/III transition $(6 ; 21-23)$. In addition to these more heuristic models, there has been an increasing use of direct two- and three-dimensional simulations of the evolving dislocation microstructures, employing the dislocation dynamics technique, in which the dislocations are the simulated entities and their movement is tracked during the simulation(11;24-28). The three-dimensional simulations are very expensive computationally, limiting the maximum stress, and the consequent dislocation density, that can be applied to the sample. Because of these limitations, direct three-dimensional dislocation simulations of strain hardening (across the range of stress and strain of interest) are not yet feasible.

The challenge is to find an approach to reduce the large number of degrees of freedom associated with typical dislocation densities to those needed to describe deformation. In other words, we need to coarse grain or homogenize the variables. One of the biggest challenges in the development of a coarse-grained theory is the presence of multiple scales. Detailed simulations (in two dimensions) show the development of a strong local order in systems of dislocations on the order of tenths of microns. $(25 ; 29 ; 30)$ Yet we know there are much bigger scales that matter in real systems. For example, dislocation cell sizes in Stage III hardened $f c c$ metals have diameters in the many tens of microns range.(4) The incorporation of both scales in a self-consistent way presents 
a huge theoretical and computational challenge that has yet to be tackled.

In this paper, we will briefly outline our coarse-graining approach based on extensions to the continuous theory of dislocations $(31 ; 32)$, discussing limitations in our approach and what questions remain. (We note that two of us recently summarized current work on coarse-graining strategies for dislocations(33)) We then will discuss how we employ simulations to address key issues in the scaling of variables as a way to link phenomena at different length and time scales.

\section{Coarse-Grained Description of Dislocation Energetics:}

We base our coarse-graining strategy on continuous dislocation theory,(34) which follows from the introduction of a coarse-graining volume $\Omega$ over which the dislocation density is averaged, as shown in Figure 2. The dislocation density is a tensor field defined at $\vec{r}$ with components $\rho_{k i}(\vec{r})$, where $k$ indicates the component of the line direction and $i$ indicates the component of the Burgers vector. The exact meaning of this averaging prescription is, however, unclear and it is not obvious at what scales a continuum theory should hold.(34)

The energy of a system of dislocations as a function of the dislocation density tensor is(35)

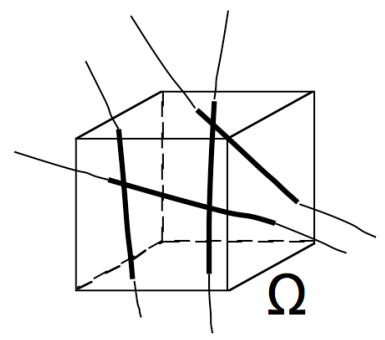

Figure 2: Averaging to form dislocation density tensor.

$E_{I}=\frac{\mu}{16 \pi} \iint \varepsilon_{i p l} \varepsilon_{j m n} R_{, m p}\left(\vec{r}, \vec{r}^{\prime}\right)\left\{\rho_{j l}(\vec{r}) \rho_{i n}\left(\vec{r}^{\prime}\right)+\delta_{i j} \rho_{k l}(\vec{r}) \rho_{k n}\left(\vec{r}^{\prime}\right)+\frac{2 v}{1-v} \rho_{i l}(\vec{r}) \rho_{j n}\left(\vec{r}^{\prime}\right)\right\} d \vec{r} d \vec{r}^{\prime}$,

where the integrals are over the entire system, $\delta_{i j}$ is the Kronecker delta, $\varepsilon_{i j k}$ is the Levi-Civita tensor, and repeated indices are summed. $R_{, m p}=\partial^{2}\left|\vec{r}^{\prime}-\vec{r}\right| / \partial x_{m} \partial x_{p}$.

While Equation (2.1) is in principle correct for continuous densities, practical applications require averaging volumes $\Omega$ that are finite. Corrections to the Kosevich form in Equation (2.1) associated with a finite averaging volume can be obtained by including higher-order moments of the dislocation density tensor in a multipolar expansion.(31) We define a set of moment densities of the distribution of loops in a volume $\Omega$, where the first two moments are

$$
\rho_{l j}^{(\Omega)}=\frac{1}{\Omega} \sum_{q=1}^{n} b_{j}^{(q)} \oint_{C^{(q)}, \Omega} d l_{l}^{(q)}, \quad \rho_{l j \alpha}^{(\Omega)}=\frac{1}{\Omega} \sum_{q=1}^{n} b_{j}^{(q)} \oint_{C^{(q)}, \Omega} r_{\alpha}^{(q)} d l_{l}^{(q)}, \ldots
$$

where the first equation is the volume-averaged dislocation density tensor (the Nye tensor), the second is the dislocation dipole density tensor, and so on. $\rho_{i j}^{(\Omega)}$ does not necessarily have the same properties as the Kröner continuous tensor, except in the limit of differential averaging volumes. Inclusion of terms that depend on the local dipole are equivalent to gradient corrections to the Kosevich form and form a natural link with strain-gradient plasticity. These expressions can be used as a basis for a continuous dislocation theory with local structure by including the dipole (and higher) dislocation moment tensors as descriptors. Numerical studies employing three-dimensional dislocation dynamics simulations have shown that the multipole expansion converges quickly and provides an accurate way to incorporate the effects of dislocation substructures.(31) 
While the general approach outlined here provides a useful way to describe coarse-grained energetics, it requires, but does not include, a detailed picture of local order and energetics. Complicating that requirement is the great divergence between local and large-scale structures, making the choice of an optimal coarse-graining length uncertain. We take the view that we will take a coarse-graining volume $\Omega$ that is large relative to local order yet small relative to the microstructural order. Given that view, we need to add to the method a description of the local ordering as well as the dynamics of that ordering. As a first step in understanding these issues, we employ a series of simple two-dimensional models.

\section{Phase-Field Modeling of Structural and Dynamic Scaling}

While we would like to employ fully-three-dimensional approaches in this work, the questions are complex enough that current three-dimensional methodologies are not practical; we cannot reach the system sizes or strain levels of interest. Thus, we restrict ourselves to two-dimensional simulations.

\subsection{The Phase-Field Method}

Our model for the complex three dimensional microstructure is a simple one. $(36 ; 37)$ We consider a system of dislocations moving on a single slip plane through a random array of forest dislocations under the action of an applied shear stress. The model is thus two-dimensional, with three dimensionality included in an approximate way by considering dislocations on other slip systems (the forest dislocations) as point obstacles to motion within the plane, ignoring all other interactions. We show a schematic view of the model system in Figure 3. While assuming a

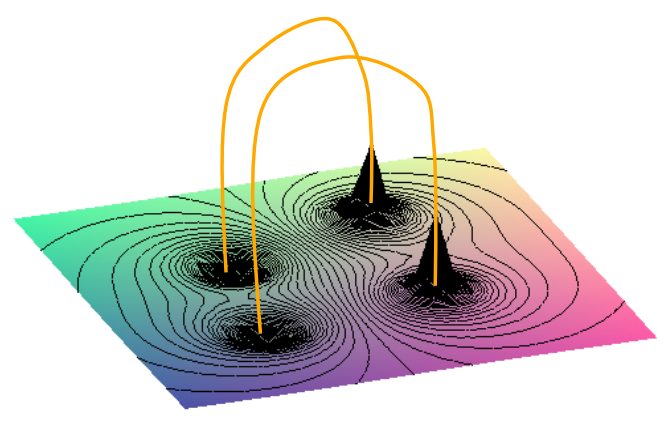

Figure 3: Schematic view of the current model, showing dislocations on a slip plane interacting with dislocations from other slip systems crossing their plane. two-dimensional model is a great simplification of a complex three-dimensional system, the predictions from the model closely match experiment.(36) We note that the assumption of point obstacles to represent the interaction with forest dislocations is a simplification and that finite-sized particles might provide a better description of those interactions. $(38 ; 39)$

Our basic approach is to develop an expression for the energetics of the system in terms of an order parameter that defines the local displacements in the system. This approach is in contrast to other methods, in which the dislocations are the key descriptors of the system. The approaches are roughly equivalent, however, in that there is a direct relationship between the displacements and the dislocation density, as will be discussed further below. The essential difference from other methods is that this approach is a continuous description; we do not identify individual, discrete, dislocations. We note that in the previous work, $(36 ; 37 ; 40)$ this approach was referred to as a 
phase-field method, largely because, as in more traditional phase-field calculations, we proceed by defining the energy of the system as a functional of some order parameter and then find the driving force by taking the functional derivative of that energy with respect to the order parameter.

The method is based on an energy expression written in terms of the plastic distortion tensor, $\beta_{m k}^{p}(x, y)$, which is then employed as the variational parameter.(40) The elastic energy in terms of $\beta_{m k}^{p}(x, y)$ is found, from which the minimum of energy and value of $\beta_{m k}^{p}(x, y)$ at the minimum is determined. The dislocation density tensor $\alpha_{i j}(x, y)$ is determined from $\beta_{m k}^{p}(x, y)$ with the relation

$$
\alpha_{j m}=\varepsilon_{i j k} \beta_{m k, j}^{p},
$$

where $\beta_{m k, j}^{p}=\partial \beta_{m k}^{p} / \partial x_{j}$.

The model assumes a set of point obstacles that represent the forest dislocations. (Terms in the energy account for dissipation when a dislocation crosses an obstacle.) An initial distribution of obstacles is assumed and then new obstacles could be added, depending on the application. As the stress increases, we measure the accumulated displacement, from which we determine the strain and dislocation content. More details are given elsewhere.(36; 37)

\subsection{Fractal Scaling of Dislocation Structures}

A measure of dislocation structure arises from the cell-size distribution having been shown (in both simulations and experiment) to be fractal $(5 ; 6)$. The indicator of a fractal system is that its descriptors can be described with power-law scaling, which arises from fractal structures being self-similar. In systems of dislocations, a convenient measure of structure is the cell size, with the probability of having a cell of size $A$ being given by

$$
n(A)=C A^{-D},
$$

where $D$ is the fractal exponent and $D^{\prime}=D-1$ is the fractal dimension. Since the fractal dimension in our model (as well as in experiments) is determined from a two-dimensional dislocation array, the 3D fractal

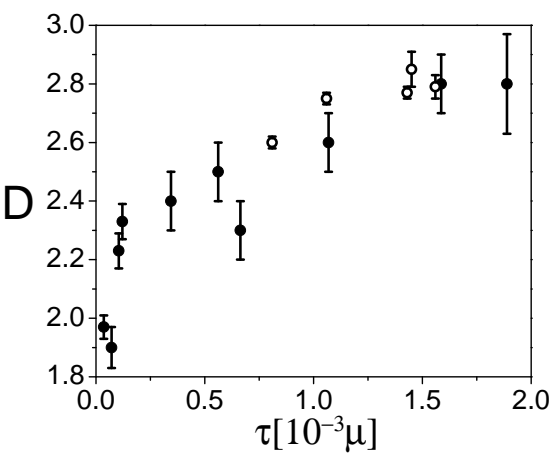

Figure 4: Fractal dimension $D$ vs. applied stress. Full circles: calculated (36); Open circles: experimental (6). dimension is obtained as $D^{\prime}+1=D$. We note that the experimental methodology for measuring cell size differs from our procedure. In the experimental studies, the walls themselves are counted, ignoring the dislocations in the cell interiors (which are not necessarily resolved in the experiment). However, if the dislocation structures are truly self-similar, as they must be if they follow Equation (3.2), then the distributions are the same regardless of the scale. We examined the development of such structures and found (in agreement with previous experiments) that the distribution of cell sizes is fractal, i.e., the structures are self-similar.(36) The number of cells of size $A$ follows the power law $N(A)=C A^{-D}$, where $D$ is the fractal dimension.(36) The calculated relationship between $D$ and applied stress agreed with experimental values, as shown in Figure 4. This relation holds over many orders in size; thus, once the distribution is known at one cell size, we have a 
measure of the structure at many others. One important issue that yet remains is to determine the scales at which the scaling law is valid.

\subsection{Intermittent Flow in Dislocation Response}

We also employed the phase-field model to examine the intermittent kinematic behavior of the dislocation response.(37) Dislocations propagate by means of avalanches; local stresses build up until a dislocation breaks free and starts an avalanche of motion of other dislocations. In our approach, dislocation loops are generated, with the net deformation proportional to the area of the loops. We found that the number of loops of area $A$ generated in an avalanche follows a power law in $A$, $N(A) \sim A^{-\sigma}$, as shown in Figure 5. We determined a power law exponent of $\sigma=1.8 \pm 0.1$, though we note that we employed a linear binning approach, which has been shown to be somewhat inaccurate.(17) Subsequent to our paper, a direct measurement of dislocation avalanches in single crystals was made and an experimental value of

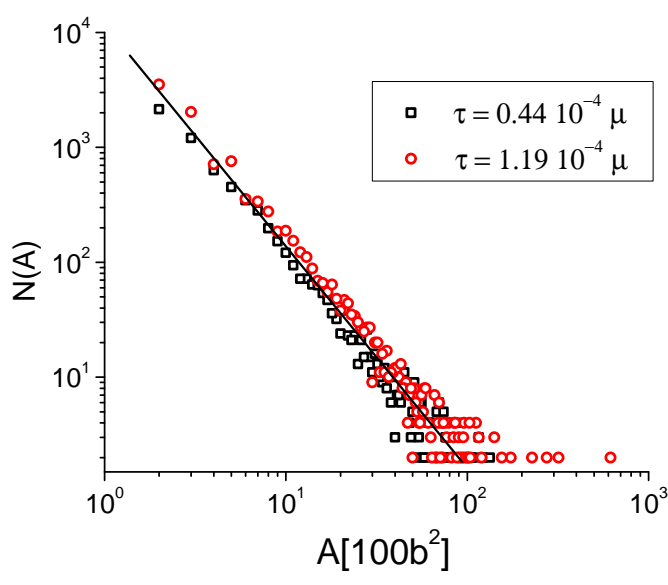

Figure 5: Probability distribution $N(A)$ at applied stress $\tau=0.44 \times 10^{-4} \mu$ and $\tau=1.19 \times$ $10^{-4} \mu$ $\sigma=1.60 \pm 0.02$ was found.(14) The latter value is in agreement with acoustic emission experiments(12) and a recent theoretical analysis.(41) The importance of these results is that they show that dislocations form a self-organized critical system, which puts them in the same class as earthquakes, fracture, etc. We can thus bring to bear on deformation another important type of scaling and analysis.

We thus have shown that dislocation structure and kinematics can be characterized by powerlaw relations over a very wide range of conditions. These scaling relations provide critical information that will form part of the basis of the development of the coarse-grained theory. These scaling relations do not, however, inform us about the details of local structural development and the balance between the structure and the noise inherent in these stochastic systems. For information about those details, we turned to an even simpler model.

\section{Energetics and Noise in Dislocation Patterning}

The ordering of dislocations into walls and other local structures takes place while the system is far from thermal equilibrium and in the presence of very large fluctuations, or noise, in the relevant variables. Noise in systems of dislocations is quite unlike the more familiar noise that arises from thermal fluctuations. In dislocations, the noise fluctuations do not constitute an independent variable in the problem, but are self consistent with the evolving configuration. In the parlance of stochastic dynamics, dislocation systems are characterized by multiplicative noise (i.e., the noise is a function of the evolving variable) rather than the additive noise found in a thermally-driven system. Employing a simple two-dimensional simulation of like-signed, parallel, edge dislocations 
on a lattice, we developed a set of quantitative functions for the energetic driving force for ordering and the resistive force owing to the noise, which exactly balance one another at the relaxed state.(42) We identified scaling relations as a function of the (scalar) dislocation density that show that the system is scale invariant, with power law dependencies of the macro-variables (energy, structure, noise) on the number of relaxing dislocations.

More specifically, we estimate the noise by determining the standard deviation of the stress at each lattice point of a slip plane, which is a measure of the fluctuations in the forces acting on a dislocation as it moves through the system. We refer to this quantity as $\mathscr{R}$, or simply the "noise." By calculating the noise over many simulations and many dislocation densities, we find that the noise is characterized by the relation

$$
\mathscr{R} \sim \rho^{1 / 2},
$$

where $\rho$ is the dislocation density, in agreement with assumptions made in an earlier work.(6)

The degree of order is derived from the standard pair correlation function for a configuration, $\mathscr{C}(x, y)$, which is the probability that a second dislocation will be found at $(x, y)$, given that the first dislocation is at $(x, y)=(0,0) .(25 ; 29)$ There is a directional dependence to $\mathscr{C}$, so that $\mathscr{C}$ does not depend simply on the scalar distance to the field dislocation. We define the differential wall correlation function $q(x)$ as the sum of $\mathscr{C}$ over the direction vertical to the slip plane,

$$
q(x)=\sum_{y}^{\prime} \mathscr{C}(x, y) .
$$

The sum is over the lattice sites in the $y$-direction, except the site at the reference dislocation, and $q(x)$ is normalized to the total number of dislocation pairs. We define an additional correlation function, $\xi_{w}$, to serve as the basis for our analysis, which we will call the total wall correlation function, or just the wall correlation function. This function represents the total excess fraction of pairs that coagulate in a central wall as the system relaxes for a given value of $\rho$. That is, for a given $\rho$, the wall correlation function is defined as the sum over $x$ of $q(x)$ for each relaxed state for which $q(x)>q($ random $)$, where $q$ (random) is the differential wall correlation function for a completely random distribution of dislocations,

$$
\xi_{w}(t, N)=\sum_{q(x)>q(\text { random })} q(x) .
$$

This function, $\xi_{w}$, is averaged over many simulations. More details are given elsewhere.(42) Analysis of the results shows

$$
\xi_{w} \sim \rho^{\alpha}
$$

where $\alpha=-0.3606 \pm .0140$, i.e., the walls become smaller as the density increases. The order is related to the noise by

$$
\xi_{w} \sim \mathscr{R}^{-0.734} .
$$

Thus we see that the higher degree of noise, the smaller the amount of order, which is in line with expectations.

Finally, we defined a driving force for formation of order for the system at fixed $\rho$ as

$$
\mathscr{F}_{\xi_{w}}(\rho)=-\left.\frac{\partial E(\rho)}{\partial \xi_{w}(\rho)}\right|_{\rho}
$$


where $E$ is the interaction energy of the system. (Complete details are given in (42).) The energy per dislocation $e(\rho)$ is found to scale as $e \propto-\mathscr{R}^{1.97}$. We find (at a time after the initial rapid relaxation)

$$
\begin{aligned}
\mathscr{F} & \sim \rho^{0.941} \\
& \sim \mathscr{R}^{1.88} .
\end{aligned}
$$

We thus demonstrated a simple linear relation between the ensemble driving force for order formation, $\mathscr{F}$, and (approximately) the square of the noise, $\mathscr{R}$, showing explicitly the balance at the relaxed state between the driving force for ordered wall formation and the frustration resistance, or noise. Beyond this central result, we find that the fully-relaxed ensemble variables are all power functions of the number of dislocations (i.e., dislocation density) in the system. Consequently, the set of ensemble variables are thus expressible as direct power functions of each other. These relations enable us to characterize the local ordering behavior of the complex dislocation system in terms of a reduced set of macrovariables.

\section{Summary and Conclusions}

We now have many components of a coarse-grained theory of dislocations, with a formalism for spatial coarse graining and scaling relations that will guide future developments. Specifically, we have found (1) the energy of a system of dislocations as function of local dislocation densities/dipoles/..., (2) scaling relations to inform on structures at scales within local volumes, (3) scaling relations for ordering (local correlations) as function of density, (4) scaling relations that inform us on the energetics of local structures (not completely discussed in this paper), and (5) scaling relations that inform us about intermittent flow. There are, however, many critical, yet unresolved, issues critical for the development of a coarse-grained theory, including the development of descriptions of order in three dimensions, descriptions of the dynamics local order, and the dynamics of the coarse-grained variables. Progress has been made in the latter case, with work by Rickman and Viñals(43), who developed an equation for the time-dependence of the dislocation density tensor based on the dislocation flux described by a Ginzburg-Landau equation, and El Azab(44), who has developed a kinetic theory of dislocations.

While much remains to be done, we are accumulating what we need for developing a theory of dislocations that will enable predictions of dislocation response without resolving all dislocations.

\section{Acknowledgments}

We thank Professor Alberto Cuitiño for the use of Figure 3. The work of R. LeSar and M. Koslowski was performed under the auspices of the United States Department of Energy (US DOE under contract W-7405-ENG-36) and was supported by the Division of Materials Science of the Office of Basic Energy Sciences of the Office of Science of the US DOE. MK thanks the Computational Materials Science Network of the US DOE/OS/OBES/DMS for their support. 


\section{References}

[1] J. Weertman and J. R. Weertman. In R. W. Cahn and P. Haasen, editors, Physical Metallurgy, volume II, pages 1259-1307, Amsterdam, 1983. North Holland.

[2] H. Mughrabi. Philosophical Magazine, 23:869-895, 1971.

[3] H. Mughrabi. Philosophical Magazine, 23:897-929, 1971.

[4] F. Szekely, I Groma, and J. Lendvai. Materials Science and Engineering A, 324:179-182, 2002 .

[5] J. Gil Sevillano, E. Bouchaud, and L. P. Kubin. Scripta Metallurgica et Materialia, 25:355360, 1991.

[6] P. Hähner, K. Bay, and M. Zaiser. Physical Review Letters, 81:2470-2473, 1998.

[7] M. Zaiser, F. M. Grasset, V. Koutsos, and E. C. Aifantis. Physical Review Letters, 93:195507, 2004.

[8] R. B. Pond. In The Inhomogeneity of Plastic Deformation, pages 1-18. American Society of Metals, 1973.

[9] N. Neuhäuser. In F. R. N. Nabarro, editor, Dislocations in Solids, volume 6, pages 319-440, Amsterdam, 1983. North Holland.

[10] J. Weiss and J. R. Grasso. Journal of Physical Chemistry B, 101:6113-6117, 1997.

[11] L. P. Kubin, C. Fressengeas, and G. Ananthakrishna. In F. R. N. Nabarro and M. S. Duesbery, editors, Dislocations in solids, volume 11, pages 101-191, Amsterdam, 2002. North Holland.

[12] M.-C. Miguel, A. Vespignani, S. Zapperi, J. Weiss, and J. R. Grasso. Nature, 410:667-671, 2001.

[13] J. Weiss and D. Marsan. Science, 299:89-92, 2003.

[14] D. M. Dimiduk, C. Woodward, R. LeSar, and M. D. Uchic. Science, 2006. in press.

[15] T. Richeton, J. Weiss, and F. Louchet. Nature Materials, 4:465-469, 2005.

[16] P Bak, C Tang, and K Wisenfeld. Physical Review Letters, 59:381-384, 1987.

[17] M. E. J. Newman. Contemporary Physics, 46:323-351, 2005.

[18] A. Turner and B. Hasegawa. ASTM, page 761, 1982.

[19] H. J. Jensen. Self-Organized Criticality. Cambridge University Press, Cambridge, UK, 1998.

[20] P. Sammonds. Nature Materials, 4:425-426, 2005.

[21] M. Zaiser. Materials Science and Engineering A, 309-310:304-315, 2001. 
[22] U. F. Kocks and H. Mecking. Progress in Materials Science, 84:171-273, 2003.

[23] R. Thomson, M. Koslowski, and R. LeSar. Physics Letters A, 332:207-212, 2004.

[24] J. Lepinoux and L. P. Kubin. Scripta Metallurgica, 21:833-838, 1987.

[25] A. N. Gulluoglu, D. J. Srolovitz, R. LeSar, and P. S. Lomdahl. Scripta Metallurgica, 23:1347$1352,1989$.

[26] N. M. Ghoniem, S.H Tong, and L.Z. Sun. Physical Review B, 61:913-927, 2000.

[27] H.M. Zbib, T. D. de la Rubia, and V. Bulatov. Journal of Engineering Materials and Technology, 124:78-87, 2002.

[28] Z. Wang, N. M. Ghoniem, R. J. McCabe, R. LeSar, and A. Misra. Acta Materialia, 52:15351542, 2004.

[29] H. Y. Wang, R. LeSar, and J. M. Rickman. Philosophical Magazine, 78:1195-1213, 1998.

[30] I. Groma, F. F. Csikor, and M. Zaiser. Acta Materialia, 51:1271-1281, 2003.

[31] R. LeSar and J. M. Rickman. Physical Review B, 69:172105, 2004.

[32] R. LeSar and J. M. Rickman. Philosophical Magazine, 83:3809-3827, 2003.

[33] J. M. Rickman and R. LeSar. Scripta Materialia, 54:735-739, 2006.

[34] E. Kröner. International Journal of Solids and Structures, 38:1115-1134, 2001.

[35] J. M. Rickman and R. LeSar. Physical Review B, 64:094106, 2001.

[36] M. Koslowski, R. LeSar, and R. Thomson. Physical Review Letters, 93:265503, 2004.

[37] M. Koslowski, R. LeSar, and R. Thomson. Physical Review Letters, 93:125502, 2004.

[38] G. Schoeck and R. Frydman. Physica Status Solidi (b), 53:661-673, 1972.

[39] S. Zapperi and M. Zaiser. Materials Science and Engineering A, 309-310:348-351, 2001.

[40] M. Koslowski, A. Cuitiño, and M. Ortiz. Journal of the Mechanics and Physics of Solids, 50:2957-2635, 2002.

[41] M. Zaiser and P. Moretti. Journal of Statistical Mechanics: Theory and Experiment, 2005:P08004, 2005.

[42] R. Thomson, M. Koslowski, and R. LeSar. Physical Review B, 73:024104, 2006.

[43] J. M. Rickman and J. Viñals. Philosophical Magazine A, 75:1251-1262, 1997.

[44] A. El-Azab. Scripta Materialia, 54:723-727, 2006. 